\title{
YM155, a selective survivin suppressant, inhibits tumor spread and prolongs survival in a spontaneous metastatic model of human triple negative breast cancer
}

\author{
KENTARO YAMANAKA, MARI NAKATA, NAOKI KANEKO, HIROSHI FUSHIKI, \\ AYA KITA, TAKAHITO NAKAHARA, HIROSHI KOUTOKU and MASAO SASAMATA \\ Drug Discovery Research, Astellas Pharma Inc., 21 Miyukigaoka, Tsukuba, Ibaraki, Japan
}

Received March 3, 2011; Accepted May 2, 2011

DOI: $10.3892 /$ ijo.2011.1077

\begin{abstract}
Metastatic triple negative breast cancer [TNBC, with negative expression of estrogen and progesterone receptors and no overexpression of HER2/neu (ErbB-2)] remains a major therapeutic challenge because of its poor overall prognosis and lack of optimal targeted therapies. Survivin has been implicated as an important mediator of breast cancer cell growth and dysfunctions in apoptosis, and its expression correlates with a higher incidence of metastases and patient mortality; thus, survivin is an attractive target for novel anticancer agents. In previous studies, we identified YM155 as a small molecule that selectively suppresses survivin expression. YM155 inhibits the growth of a wide range of human cancer cell lines. Tumor regression induced by YM155 is associated with decreased intratumoral survivin expression, increased apoptosis and a decreased mitotic index. In the present study, we evaluated the antitumor efficacy of YM155 both in vitro and in vivo using preclinical TNBC models. We found that YM155 suppressed survivin expression, including that of its splice variants (survivin $2 \mathrm{~B}, \delta \mathrm{Ex} 3$ and $3 \mathrm{~B}$ ), resulting in decreased cellular proliferation and spontaneous apoptosis of human TNBC cells. In a mouse xenograft model, continuous infusion of YM155 led to the complete regression of subcutaneously established tumors. Furthermore, YM155 reduced spontaneous metastases and significantly prolonged the survival of animals bearing established metastatic tumors in the MDA-MB-231Luc-D3H2-LN orthotopic model. These results suggest that the survivin-suppressing activity of YM155 may offer a novel therapeutic option for patients with metastatic TNBC.
\end{abstract}

\section{Introduction}

Breast cancer is one of the leading causes of cancer death worldwide (1). For patients with local disease, breast cancer is

Correspondence to: Dr Kentaro Yamanaka, Drug Discovery Research, Astellas Pharma Inc., 21 Miyukigaoka, Tsukuba, Ibaraki 305-8585, Japan

E-mail: kentaro.yamanaka@jp.astellas.com

Key words: YM155, survivin suppressant, breast cancer, metastasis potentially curable by surgical resection combined with adjuvant therapy, including radiation, anti-estrogen therapy and Her2targeting agents. However, management of metastatic breast cancer is far less successful than treatment of local disease. Despite the recent progress in molecular-targeted therapies, metastatic breast cancer remains the principal cause of breast cancer death and is a major therapeutic challenge for selecting optimal treatment (2).

Recent molecular characterization of genetic signatures for patient stratification revealed that triple negative breast cancer (TNBC), with negative expression both of estrogen and progesterone receptors and no overexpression of the Her2 protein, is a high-risk factor with limited therapeutic options. Retrospective studies suggest that TNBC patients represent $\sim 15 \%$ of total breast cancers, and that this cancer type may be more aggressive, with rapid tumor growth, a high incidence of metastasis, an increased possibility of distant recurrence, and a higher mortality rate than other breast cancers (3-5). Metastatic TNBC patients have a higher response rate than patients with hormone receptor-positive breast cancer at the initial stage of chemotherapy $(6,7)$. However, the median overall survival rate is only 1 year (8). Thus, there is a pressing need to develop novel therapeutic options that are suitable for this subgroup of patients.

Survivin is a member of the inhibitor of apoptosis (IAP) family of proteins. Increased survivin expression is observed in breast cancer cells, while no survivin transcripts are detectable in normal breast tissue. Retrospective analyses revealed that up-regulation of survivin correlates with decreased survival rates, increased relapse, and a higher frequency of metastases in breast cancer patients (9-11). Also, survivin is highly expressed in most cancer cell lines, where its expression confers resistance to apoptosis induced by various chemotherapeutic agents $(12,13)$. Inhibition of survivin using ribozyme and a dominant-negative mutant induced spontaneous apoptosis and decreased the growth rate of breast cancer cells $(14,15)$. A recent study suggested that upregulation of survivin (activated by Notch) preferentially promotes apoptosis dysfunction in human TNBC cells and plays an important role in the aggressive nature of this disease (16). Considering its preferential expression in cancer cells, its ability to confer aggressive tumor behavior, and its correlation with poor patient 
prognosis, survivin stands out as a promising target for cancer therapy.

In previous studies, we identified the small molecule (YM155) that suppresses survivin expression using a high through-put screening assay and a truncated survivin promoter. We found that YM155 selectively suppresses survivin expression without affecting other IAP proteins, resulting in apoptosis induction in hormone refractory prostate cancer cells (17). In vitro large panel screening revealed that YM155 has nanomolar antiproliferative activity against a broad range of human cancer cell lines (18). TNBC cells were among the top three sensitive cell lines. In the in vivo setting, continuous infusion of YM155 induces tumor regression and decreases intratumoral survivin expression, increases apoptosis, and decreases the mitotic index (19). These results underscore the need for extensive investigation of the role of YM155 in breast cancer treatment and to develop this compound as a novel therapeutic option for metastatic breast cancer patients.

Given the fact the majority of breast cancer deaths are caused by metastatic disease in regional and distant sites, such as lymph nodes, lung, liver, brain and bone, therapeutic options that inhibit metastatic dissemination would be important additions to the arsenal of drugs in clinical use. In the present study, we evaluated the in vitro antitumor efficacy of YM155 against TNBC cells. We also examined the in vivo efficacy of YM155 on a subcutaneously established xenograft tumor and in a spontaneous metastases model of human TNBC cancer cells.

\section{Materials and methods}

Cell culture. The human breast cancer cell line, MRK-nu-1 (JCRB0628), was obtained from Japanese Collection of Research for Bioresources. The cells were cultured at $37^{\circ} \mathrm{C}$ in a $5 \% \mathrm{CO}_{2}$ atmosphere in RPMI-1640 medium supplemented with $10 \%$ heat-inactivated fetal bovine serum (FBS). The Bioware ${ }^{\circledR}$ cell line, MDA-MB-231-Luc-D3H2-LN, was derived from MDA-MB-231 human mammary gland adenocarcinoma cells by stable transfection of the North American firefly luciferase gene, the expression of which is driven by the SV40 promoter. MDA-MB-231-Luc-D3H2-LN cells were cultivated in MEM supplemented with L-glutamine, non-essential amino acids, sodium pyruvate and $10 \%$ FBS.

Reagents. YM155 monobromide (YM155) was synthesized by Astellas Pharma Inc. (Tokyo, Japan). For the in vitro experiments, test compounds were dissolved in dimethyl sulfoxide (DMSO) and diluted with culture medium [final concentration of DMSO in culture medium: $0.1 \%(\mathrm{v} / \mathrm{v})]$. For in vivo studies, the dose of YM155 was expressed as that of the cationic moiety of the drug. Docetaxel (Taxotere ${ }^{\circledR}$ injection) was purchased from Sanofi Aventis Co., Ltd. (Bridgewater, NJ, USA). All drugs were dissolved and diluted in saline just before administration.

In vitro assay for cell viability and caspase activity. Cell viability or caspase activity was determined using a CellTiter-Glo ${ }^{\circledR}$ luminescent cell viability assay or a Caspase-Glo3 $/ 7^{\circledR}$ luminescent assay (Promega, Madison, WI, USA). The luminescence of each sample was measured in a plate-reading luminometer (EnVision ${ }^{\circledR}$; Perkin-Elmer, Waltham, MA, USA) using a 1-min lag time and $0.5 \mathrm{sec} /$ well read time. The luminescent signals for the drug-treated cells were normalized to that of the control. The assay was performed in triplicate, and the mean value was obtained based on the results of three independent assays.

Total RNA preparation, cDNA synthesis and quantitative real-time PCR. Total RNA was isolated from the cells using an RNeasy mini kit (Qiagen, CA, USA) according to the manufacturer's instructions. cDNA was synthesized from $250 \mathrm{ng}$ of total RNA primed with random hexamers using High Capacity RNA-to-cDNA Master Mix (Life Technologies Corporation, CA, USA). Real-time PCR was performed using the ABI Prism 7900 sequence detection system and Power SYBR-Green PCR Master Mix (Applied Biosystems, CA, USA) according to the manufacturer's instructions. The following primer sequences were designed using the Primer Express software (Applied Biosystems): human survivin 5'-CTGCCTGGCAGCCCTTT-3' (forward) and 5'-CCTCC AAGAAGGGCCAGTTC-3' (reverse); human survivin 2B 5'-CGCCTGTAATACCAGCACTTTG-3' (forward) and 5'-GACGAATGCTTTTTATGTTCCTCTCT-3' (reverse); human survivin $\delta E x 3$ 5'-CCAGAGTGGCTGCACCACT TCC-3' (forward) and 5'-ACAGGAAGGCTGGTGGCACC-3' (reverse); human survivin 3B 5'-GGGACTGGAAGCAAAAG AATTTC-3' (forward) and 5'-TCCTCTTCATGTCCAGTTAA ACAGA-3' (reverse); human 18S rRNA 5'-GCCGCTAGAGGT GAAATTCTTG-3' (forward) and 5'-CATTCTTGGCAAAT GCTTTCG-3' (reverse). Dissociation curve analyses were done to verify that there was no non-specific amplification or formation of primer dimers. Values were calculated based on standard curves generated for each gene. Normalization of samples was determined by dividing the number of copies of survivin transcripts by the number of copies of $18 \mathrm{~S}$ rRNA. All sets of reactions were conducted in triplicate. The relative expression levels were expressed as a percentage of the indicated control.

Western blot analysis. Protein was extracted using RIPA buffer containing protease and phosphatase inhibitors. Proteins $(20 \mu \mathrm{g})$ were resolved on SDS-PAGE gels and transferred onto PVDF membranes. After blocking at room temperature with TBS-T buffer containing 10\% Blocking One (Nacalai Tesque, Kyoto, Japan), each membrane was incubated overnight at $4^{\circ} \mathrm{C}$ with the following primary antibodies: affinity-purified rabbit anti-survivin, rabbit-cleaved PARP (Cell Signaling, Beverly, MA, USA) or rabbit anti-actin (Sigma-Aldrich, MO, USA). After washing the membranes with TBS-T, they were incubated with an HRP-conjugated secondary antibody for $1 \mathrm{~h}$ at room temperature. Proteins of interest were visualized by enhanced chemiluminescence using ECL (Amersham, Arlington Heights, IL, USA).

MRK-nu-1 mouse xenograft model. Animal experiments were performed in accordance with strict institutional guidelines and the approval of the internal committee at Astellas Pharma Inc. MRK-nu-1 cells were mixed with Matrigel ${ }^{\circledR}$ (BectonDickinson Co., Franklin Lakes, NJ, USA) and $3 \times 10^{6}$ cells were subcutaneously injected into the flanks of five-week-old 
(A)
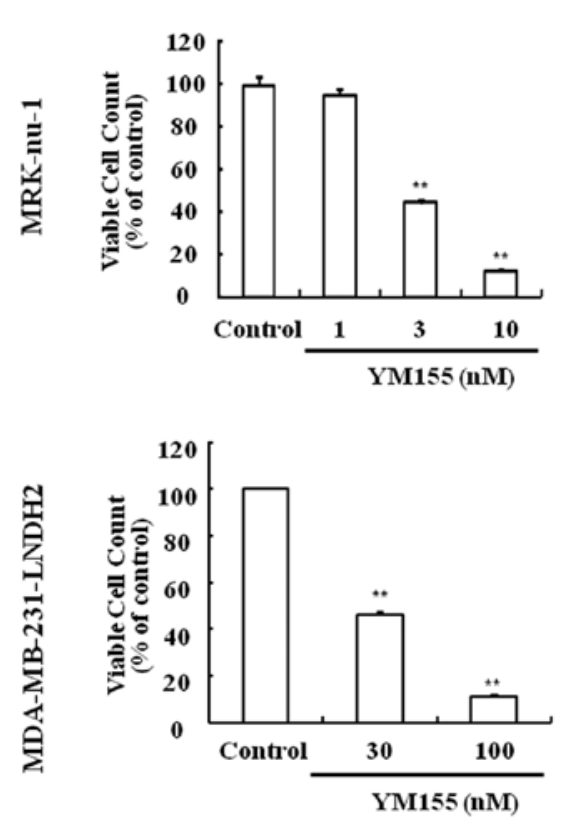

(B)
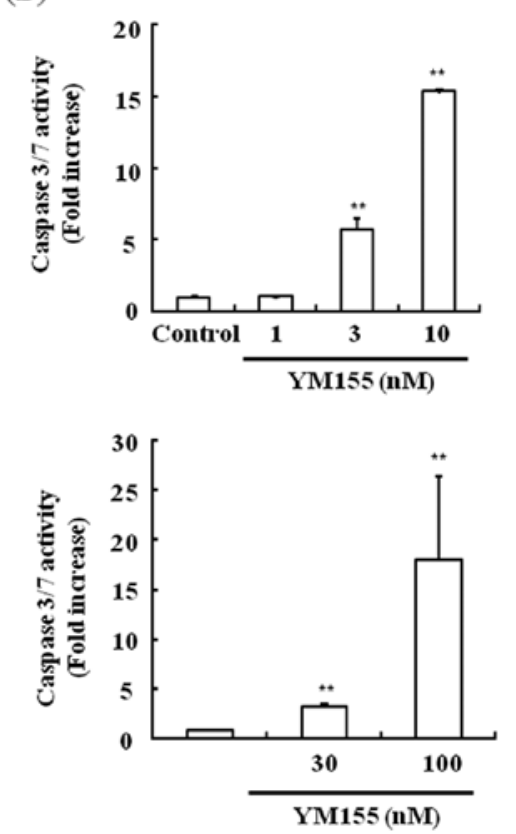

Figure 1. Effect of YM155 on cell viability, and caspase-3 and -7 activities in the MRK-nu-1 and MDA-MB-231-Luc-D3H2-LN human TNBC cell line. Each bar represents the mean \pm SE from three separate experiments. Cells were treated with the indicated concentrations of YM155 for $24 \mathrm{~h}$. ** $\mathrm{p}<0.01$, Dunnett's test. (A) Viable cell counts were normalized to that of control cells and expressed as \% of control. (B) Caspase activities were normalized to cell number and expressed as the -fold increase over the control.

female nude mice, CAnN.Cg-Foxn1nu/CrlCrlj (nu/nu). After the tumors were established, mice were divided into treatment groups based on tumor volume to minimize intragroup and intergroup differences in tumor volume and body weight. The first day of administration was designated as day 0 , and observation continued until day 21. YM155 was administered as a 7-day continuous infusion using a micro-osmotic pump (Alzet $^{\circledR}$ model 1003D; Durect, Cupertino, CA, USA). Body weight and tumor diameter were measured once a week, and tumor volume was determined (length $\mathrm{x}$ width ${ }^{2} \mathrm{x} 0.5$ ). Complete regression $(\mathrm{CR})$ was defined as tumor regression to below the limit of palpation.

MDA-MB-231-luc mouse metastasis model from orthotopically implanted mammary gland tumors. Nine-week-old CB17 SCID females were anesthetized and $20 \mu \mathrm{l}$ of $1 \times 10^{5}$ MDA-MB-231-Luc-D3H2-LN cells were injected into the mammary gland. Six weeks later, the primary mammary gland tumor was surgically removed, and the incision was closed with surgical sutures and tissue glue under anesthesia. Metastatic tumor growth was monitored by in vivo bioluminescent imaging of the chest area using IVISSpectrum, with the primary tumor shielded (Caliper Life Sciences, Hopkinton, MA). The measurements were performed weekly from the time of primary tumor resection, and then twice weekly until the end of the study. The treatment was initiated when detectable metastases developed in the chest area as determined by biophotonic imaging. Mice were divided into three groups to minimize intragroup and intergroup variations in average bioluminescence. The first day of drug treatment was designated as day 0 , and observation continued until day 32 , when $>50 \%$ of the mice from the group treated with YM155 were euthanized. During the study, the body weight and general health of the mice were monitored daily. Mice were scored as dead if any of the following signs of suffering occured: cachexia, weakening, or difficulty moving or eating. Axillary and mesenteric lymph nodes, lung, liver, brain and femurs were dissected from representative mice and evaluated by in vivo bioluminescence imaging.

Statistical analysis. Values were expressed as the mean $\pm \mathrm{SE}$ of the mean (SEM). For the in vitro studies, Dunnett's test was used for the comparison and determination of statistical significance between the control and each YM155-treated group. For the in vivo study, tumor volume and body weight between the control and YM155-treated group on day 21 were compared using the Student's t-test. For in vivo biophotonic imaging, statistical analysis was performed using the MannWhitney U-test. For survival analysis, Kaplan-Meier curves were generated and the differences between the control and each drug-treated group were assessed using the log-rank test. All data analyses were performed using SAS software (SAS Institute, Cary, NC, USA) and $\mathrm{p}<0.05$ was to be considered statistically significant.

\section{Results}

YM155 inhibits cancer cell growth and activates caspase-3 and -7 in human TNBC cells in vitro. We first evaluated the in vitro effect of YM155 on cancer cell growth and caspase activity in human TNBC cell lines. MRK-nu-1 and MDA-MB231-Luc-D3H2-LN cells were treated with YM155 (1-100 nM). At $24 \mathrm{~h}$ post treatment, YM155 significantly decreased the viable cell count by $>50 \%$ compared with control cells treated with DMSO in both cell lines (Fig. 1A). When exposed to YM155, both TNBC cell lines showed a dose-dependent 

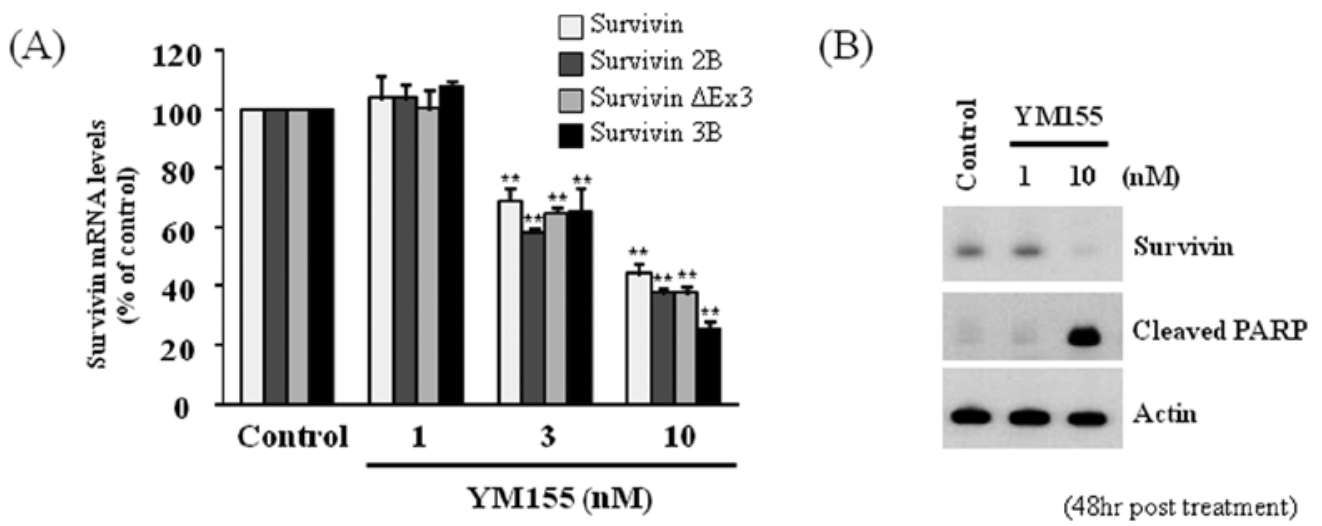

Figure 2. Effect of YM155 on survivin mRNA expression and protein expression in the MRK-nu-1 human TNBC cell line. Each bar represents the mean \pm SE from three separate experiments. Cells were treated with the indicated concentrations of YM155 for $48 \mathrm{~h}$. (A) Expression levels of survivin mRNA $48 \mathrm{~h}$ posttreatment were normalized to that of $18 \mathrm{~S}$ rRNA and expressed as relative expression levels (\% of control). (B) Protein expression levels $48 \mathrm{~h}$ post-treatment were determined by Western blotting with the indicated antibodies.
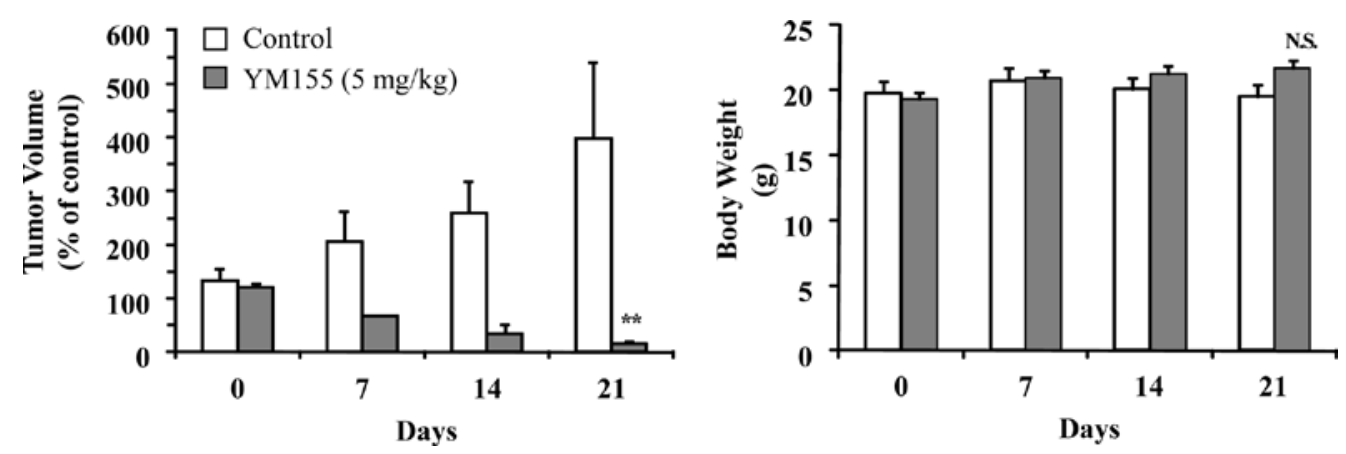

Figure 3. In vivo antitumor activity of YM155 in s.c. xenografted MRK-nu-1 human TNBC tumors. YM155 was administered as a 7-day continuous infusion. Values are expressed as the mean \pm SE $(n=5)$. Tumor volume and body weight in the control and YM155 groups on day 21 were statistically evaluated using the Student $\mathrm{t}$-test; ${ }^{* *} \mathrm{p}<0.01$ and N.S. (not significant) versus control. Complete regression (CR) was defined as tumor regression to below the limit of palpation.

increase in caspase-3 and -7 activity (Fig. 1B). These results indicated that YM155 had nanomolar antiproliferative activity against human TNBC cells and triggered the induction of spontaneous apoptosis in vitro.

YM155 down-regulates survivin expression and induces spontaneous apoptosis in human TNBC cells in vitro. We next examined the in vitro effect of YM155 on endogenous survivin expression and apoptosis in the MRK-nu-1 cell line. YM155 treatment at 3 and $10 \mathrm{nM}$ for $48 \mathrm{~h}$ significantly suppressed survivin mRNA expression in a dose-dependent manner. Similarly, suppressed expression of the mRNA for the survivin splice variants survivin-2B, $\delta E x 3$ and $3 B$ was also observed (Fig. 2A). Western blot analysis confirmed that YM155 treatment decreased the level of survivin protein and induced an increase in the amount of cleaved poly ADP-ribose polymerase. No apparent change was observed in the expression of actin (Fig. 2B). These results indicated that YM155 abrogated endogenous survivin expression both at the mRNA and protein level, and induced spontaneous apoptosis in human MRK-nu-1 cells.

YM155 induces tumor regression in subcutaneously established human TNBC xenograft tumors. The in vivo antitumor activity of YM155 was evaluated in established MRK-nu-1 subcutaneously xenografted mice. YM155 was administered as a 7-day infusion once every 3 weeks at $5 \mathrm{mg} / \mathrm{kg} /$ day $(\mathrm{n}=5)$. The continuous infusion of YM155 completely inhibited tumor growth and induced marked regression of established tumors. Two out of five mice experienced complete tumor regression, while continued tumor growth was observed in the control group. Mice administered YM155 infusions showed no signs of tumor regrowth or significant body weight loss during the observation period (Fig. 3). These results suggested that YM155 induced marked tumor regression in established human TNBC xenograft tumors without any evidence of systemic toxicity.

YM155 decreases in vivo tumor spread and prolongs survival in a spontaneous metastatic model. The in vivo antitumor efficacy of YM155 was evaluated in the MDA-MB-231-Luc-D3H2-LN mouse metastasis model using an orthotopic mammary gland tumor. After cell implantation in the mammary gland of female nude mice, all mice developed primary tumors after mammary gland tumor resection (Fig. 4A). YM155, or a vehicle control, was administered as a 7-day infusion at $2 \mathrm{mg} /$ $\mathrm{kg} / \mathrm{day}$. A single dose of docetaxel $(15 \mathrm{mg} / \mathrm{kg})$, or a vehicle control, was administered intravenously on day 0 . On day 14 
(A)

Cell injection Mastectomy

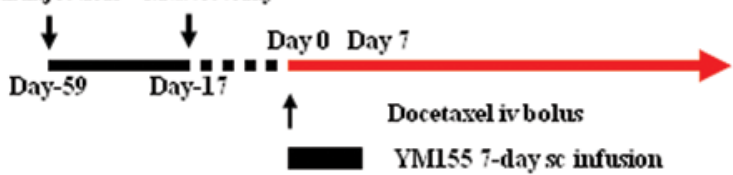

$\longrightarrow$ Primary tumor growth

(C)

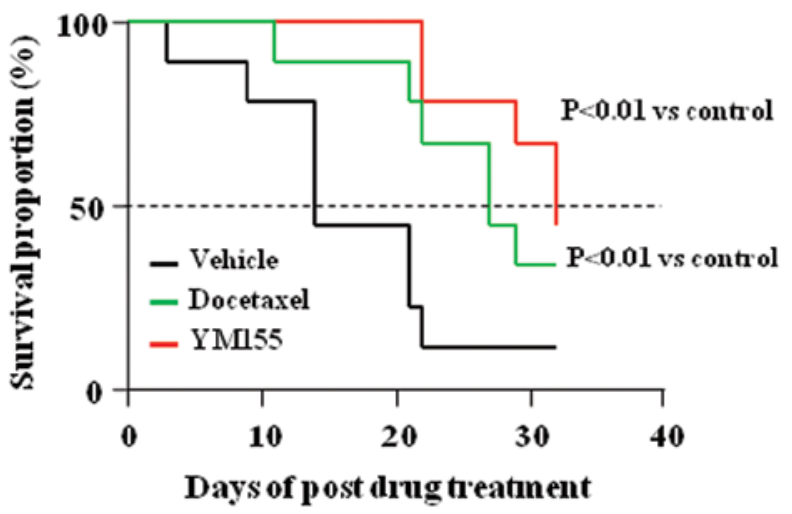

(B)
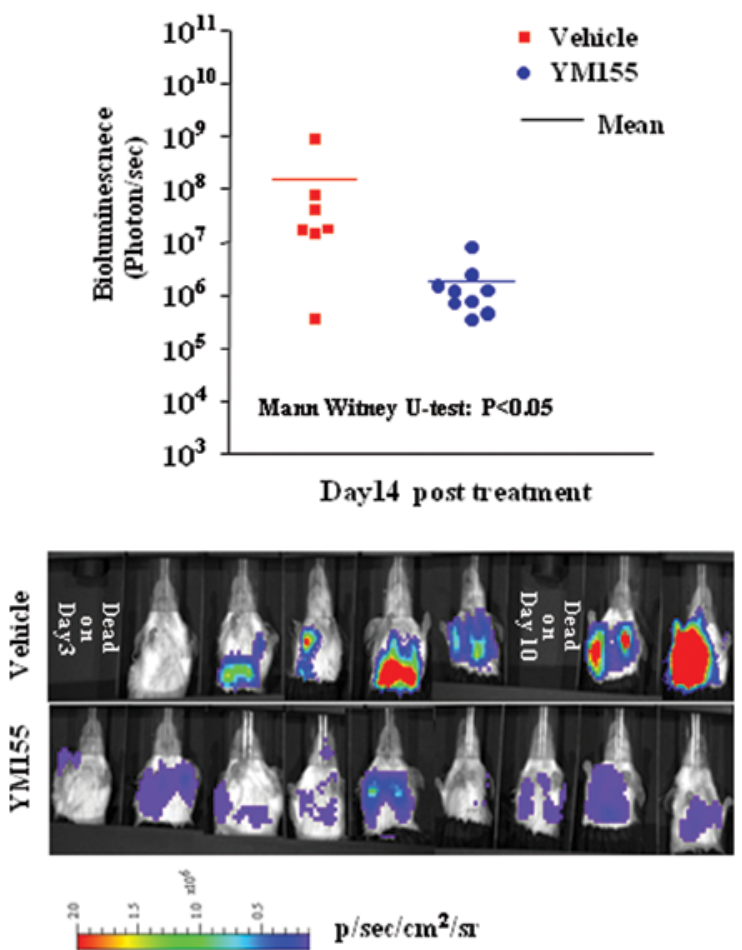

Figure 4. In vivo antitumor efficacy of YM155 in the MDA-MB-231-Luc-D3H2-LN human TNBC orthotopic metastasis model. (A) Experimental protocol for the MDA-MB-231-Luc-D3H2-LN orthotopic tumor metastasis study. (B) The metastatic tumor growth on day 14 was monitored by in vivo bioluminescent imaging of the chest area. A pseudocolor scale bar was used to obtain relative changes at metastatic sites for all images of dorsal views. $p<0.05$ versus vehicle control, Mann-Whitney U-test. (C) Kaplan-Meier plot of \% survival in control- (vehicle), docetaxel- and YM155-treated groups. p $<0.01$ versus vehicle control, log-rank test.

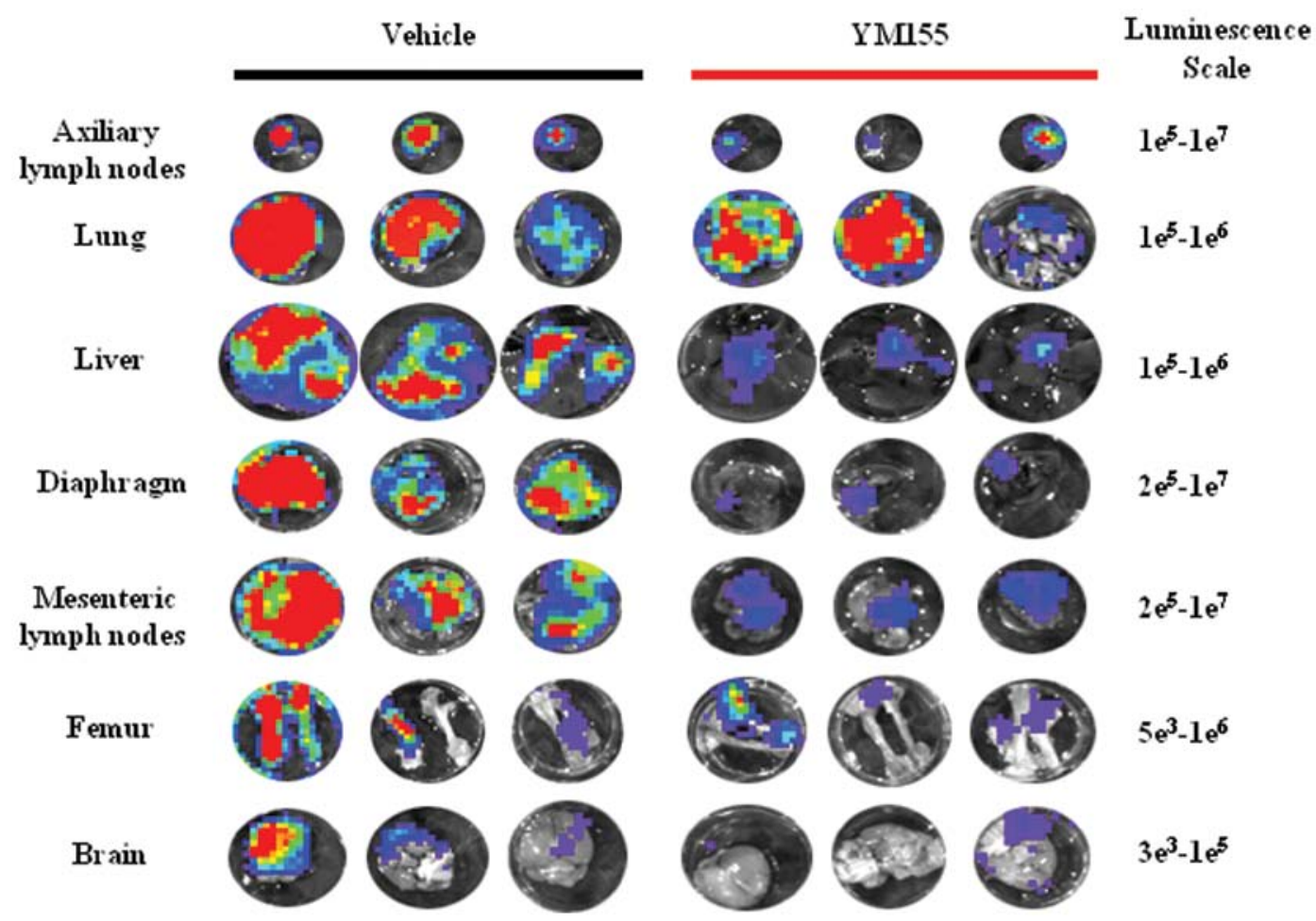

Figure 5. The effect of YM155 on multiple spontaneous metastases visualized by bioluminescent imaging. The major metastatic tissues, including the axillary lymph nodes, lung, liver, mesenteric lymph nodes, brain and femurs were dissected at the time of euthanasia and imaged using ex vivo bio-imaging. The pseudocolor scale bars were consistent for all images and show relative changes at metastatic sites over time. 
post-treatment, the mean bioluminescence in the groups treated with YM155 was clearly lower than that in the vehicletreated group, indicating that YM155-treated animals tended to exhibit fewer total chest metastases than control animals and a propensity toward reduced metastasis (Fig. 4B). Metastasis-related mortality began on day 3 in the vehicletreated control mice. The median survival times were 14 days in the vehicle-treated control group, 27 days in the docetaxel treated group, and 32 days in the YM155 treated group. YM155 and docetaxel significantly prolonged the survival of animals bearing metastatic tumors compared with the vehicle-treated group (Fig. 4C). These results showed that YM155 reduced the frequency of metastasis and prolonged survival in a spontaneous metastasis mouse model of TNBC breast cancer.

Analysis of metastatic tumor growth in representative mice by ex vivo bioluminescent imaging of tissues. Given the fact that breast cancer frequently spreads to the axillary lymph nodes, bone, lung, liver or brain, we further conducted ex vivo imaging to clarify the inhibitory effects of YM155 on MDAMB-231-Luc-D3H2-LN metastatic tumors. Qualitative analysis of the bioluminescent imaging results obtained from the axillary lymph nodes, lung, liver, diaphragm, mesenteric lymph nodes, brain and femurs (Fig. 5). Bioluminescent imaging confirmed that the metastatic tumor burden in mice treated with YM155 was apparently lower than that of vehicle-treated controls. These results indicated that MDA-MB-231-Luc-D3H2-LN tumors are aggressively metastatic and intra-vascularly invade the surrounding tissues to establish metastases. Also, these results suggested that YM155 treatment inhibited the development of metastases and blocked tumor cell dissemination, even after metastases were established.

\section{Discussion}

In the present study, we evaluated the therapeutic potential of YM155 in a preclinical human TNBC model both in vitro and in vivo. We found that YM155 exhibited nanomolar antiproliferative activity and induced apoptosis in human TNBC cell lines in vitro. YM155 also downregulated the expression of survivin mRNA and protein levels. RT-PCR revealed that YM155 suppressed the expression of survivin splice variants, including survivin $2 \mathrm{~B}, \delta \mathrm{Ex} 3$, and $3 \mathrm{~B}$ in human TNBC cells. YM155-induced survivin suppression was accompanied by the induction of spontaneous apoptosis. In the MRK-nu-1 mouse xenograft model, continuous infusion of YM155 induced complete tumor regression of subcutaneously established TNBC tumors without any decrease in body weight. Furthermore, we demonstrated that YM155 reduced spontaneous metastases and significantly prolonged the survival of animals bearing established metastatic tumors derived from the human TNBC cell line, MDA-MB-231-Luc-D3H2-LN.

Aberrant cell proliferation and apoptosis resistance are hallmarks of cancer cells (20). Survivin is an intriguing protein that facilitates tumor growth, inhibits cancer apoptosis, and functions as an effector protein downstream of Her2 signaling in breast cancer $(12,13,21)$. Given its prominent expression in human breast cancer and low expression (or absence) in most normal tissues, survivin is an ideal target for mono- or combination cancer therapy. Here, we showed that YM155 exhibits nanomolar antiproliferative activity against human TNBC cells (Fig. 1). Consistent with the fact that YM155 was identified as a selective survivin transcriptional inhibitor using a truncated promoter region, YM155 suppressed mRNA expression levels of survivin, including the splice variants survivin-2B, $-\delta E x 3$, and $-3 B$ (Fig. 2). To date, four survivin variants have been described: survivin- $2 \alpha,-2 \beta,-\delta E x 3$ and $-3 B$ (22). Survivin $\delta E x 3$ is anti-apoptotic (23), whereas $2 \alpha$ and $2 \mathrm{~B}$ are potentially pro-apoptotic $(24,25)$. Although it remains unclear whether these splicing variants mediate apoptosis inhibition or induction in TNBC cells, spontaneous apoptosis induced by YM155 is accompanied with the suppression of wild-type survivin and its splicing variants (Fig. 2). The correlation between expression of survivin splice variants and prognostic significance has been studied in patients with breast cancer. These studies demonstrated a correlation between survivin and/or splice variant expression, the presence of mutant p53, and the absence of estrogen and progesterone receptors. It is also known that wild-type survivin is the most dominant transcript in breast tumor biopsies $(26,27)$. Taken together, therapeutic targeting of the survivin pathway may be beneficial for the treatment of TNBC patients.

Metastasis is the terminal event of cancer development and the principal cause of mortality in patients with breast cancer. Also, cancer metastasis is often attributed to the complex and multiphase nature of cancer development, including invasion into the local surrounding tissues, entry into the circulatory or lymphatic systems, and transport of the metastatic cells, which results in the development of distant micrometastasis. Given these phenotypic changes, breast cancer cells allegedly acquire several genetic changes, including apoptosis resistance, as the primary tumor progresses toward metastasis (28). Consistent with this, chemotherapy of metastatic breast cancer is far less successful than treatment of the primary disease $(5,29)$. Particularly for TNBC, poor prognosis and a lack of therapeutic agents has increased the demand for novel therapeutic options.

Recently, it was found that estrogen receptor-negative breast cancer cells preferentially show Notch-mediated survivin upregulation, which promotes an inherent resistance to apoptosis (16). In the present study, YM155 induced tumor regression with negligible systemic toxicity as evidenced by an absence of body weight loss (Fig. 3). Furthermore, we provide the first evidence that YM155 may have therapeutic value in reducing the spontaneous metastasis of human TNBC cells in vivo (Figs. 4 and 5). Considering the fact that survivin is overexpressed in high grade invasive and metastatic human tumors, it is suggested that dysregulation of survivin expression may confer an ability to evade immune responses and physical barriers to invasion of normal tissues. Conversely, breast cancer cells may remain sensitive to apoptosis induction when survivin is downregulated by YM155, even after distant metastasis have been established that may have accumulated additional genetic changes and new blood vessels that nourish tumor growth.

YM155 protected animals with established metastases and resulted in a significant survival benefit (Fig. 4). We used docetaxel, which is effective for the treatment of metastatic 
breast cancer irrespective to HER2 status, as the reference compound. In the clinical setting, a reduction in metastasis is expected to improve the quality of life and increase survival rates. The spontaneous metastatic model is a better representation of the true clinical situation than subcutaneous tumors, and offers persuasive evidence for the clinical development of YM155 as a novel therapeutic option in breast cancer. Further studies will be worthwhile to establish the best form of combination therapy and to identify individuals most likely to respond to YM155-based therapy.

In conclusion, our data show that survivin is a potential target for breast cancer treatment, and that YM155 may be a promising therapeutic agent for the treatment of patients with metastatic TNBC. As a novel survivin suppressor, further clinical investigation of YM155 is worthwhile. A phase II open-label study of YM155 in combination with docetaxel in patients with breast cancer is currently underway.

\section{Acknowledgment}

We thank Dr Olesia Buiakova and Dr Yan Xu (Taconic Farms, Inc., Cranbury, NJ) for in vivo bioimaging study, and members of our project team at Astellas Pharma Global Development for their constructive comments on the manuscript. This study was funded by Astellas Pharma, Inc., Japan.

\section{References}

1. Jemal A, Siegel R, Xu J and Ward E: Cancer statistics. CA Cancer J Clin 60: 277-300, 2010.

2. Guameri V and Conte P: Metastatic breast cancer: therapeutic options according to molecular subtypes and prior adjuvant therapy. Oncologist 14: 645-656, 2009.

3. Conlin AK and Seidman AD: Beyond cytotoxic chemotherapy for the first-line treatment of HER2-negative, hormoneinsensitive metastatic breast cancer: current status and future opportunities. Clin Breast Cancer 8: 215-223, 2008.

4. Liedtke C, Mazouni C, Hess KR, André F, Tordai A, Mejia JA, Symmans WF, Gonzalez-Angulo AM, Hennessy B, Green M, Cristofanilli M, Hortobagyi GN and Pusztai L: Response to neoadjuvant therapy and long-term survival in patients with triple-negative breast cancer. J Clin Oncol 26: 1275-1281, 2008.

5. Dent R, Trudeau M, Pritchard KI, Hanna WM, Kahn HK, Sawka CA, Lickley LA, Rawlinson E, Sun P and Narod SA: Triple-negative breast cancer: clinical features and patterns of recurrence. Clin Cancer Res 13: 4429-4434, 2007.

6. Rouzier R, Perou CM, Symmans WF, Ibrahim N, Cristofanilli M, Anderson K, Hess KR, Stec J, Ayers M, Wagner P, Morandi P, Fan C, Rabiul I, Ross JS, Hortobagyi GN and Pusztai L: Breast cancer molecular subtypes respond differently to preoperative chemotherapy. Clin Cancer Res 11: 5678-5685, 2005.

7. Parker JS, Mullins M, Cheang MC, Leung S, Voduc D, Vickery T, Davies S, Fauron C, He X, Hu Z, Quackenbush JF, Stijleman IJ, Palazzo J, Marron JS, Nobel AB, Mardis E, Nielsen TO, Ellis MJ, Perou CM and Bernard PS: Supervised risk predictor of breast cancer based on intrinsic subtypes. J Clin Oncol 27: 1160-1167, 2009.

8. Kassam F, Enright K, Dent R, Dranitsaris G, Myers J, Flynn C, Fralick M, Kumar R and Clemons M: Survival outcomes for patients with metastatic triple-negative breast cancer: implications for clinical practice and trial design. Clin Breast Cancer 9: 29-33, 2009.

9. Nasu S, Yagihashi A, Izawa A, Saito K, Asanuma K, Nakamura M, Kobayashi D, Okazaki M and Watanabe N: Survivin mRNA expression in patients with breast cancer. Anticancer Res 22: 1839-1843, 2002

10. Dogu GG, Ozkan M, Ozturk F, Dikilitas M, Er O and Ozturk A: Triple-negative breast cancer: immunohistochemical correlation with basaloid markers and prognostic value of survivin. Med Oncol 27: 34-39, 2010.
11. Tanaka K, Iwamoto S, Gon G, Nohara T, Iwamoto $M$ and Tanigawa N: Expression of survivin and its relationship to loss of apoptosis in breast carcinomas. Clin Cancer Res 6: 127-134, 2000.

12. Altieri DC: Opinion - survivin, cancer networks and pathwaydirected drug discovery. Nat Rev Cancer 8: 61-70, 2008.

13. Altieri DC: Survivin and IAP proteins in cell-death mechanisms. Biochem J 430: 199-205, 2010.

14. Choi KS, Lee TH and Jung MH: Ribozyme-mediated cleavage of the human survivin mRNA and inhibition of antiapoptotic function of survivin in MCF-7 cells. Cancer Gene Ther 10: 87-95, 2003.

15. Mesri M, Wall NR, Li J, Kim RW and Altieri DC: Cancer gene therapy using a survivin mutant adenovirus. J Clin Invest 108: 981-990, 2001 .

16. Lee CW, Raskett CM, Prudovsky I and Altieri DC: Molecular dependence of estrogen receptor-negative breast cancer on a Notch-survivin signaling axis. Cancer Res 68: 5273-5281, 2008.

17. Nakahara T, Takeuchi M, Kinoyama I, Minematsu T, Shirasuna K, Matsuhisa A, Kita A, Tominaga F, Yamanaka K, Kudoh M and Sasamata M: YM155, a novel small-molecule survivin suppressant, induces regression of established human hormone-refractory prostate tumor xenografts. Cancer Res 67: 8014-8021, 2007.

18. Nakahara T, Kita A, Yamanaka K, Mori M, Amino N, Takeuchi M, Tominaga F, Kinoyama I, Matsuhisa A, Kudou M and Sasamata M: Broad spectrum and potent antitumor activities of YM155, a novel small-molecule survivin suppressant, in a wide variety of human cancer cell lines and xenograft models. Cancer Sci 102: 614-621, 2011.

19. Nakahara T, Yamanaka K, Hatakeyama S, Kita A, Takeuchi M, Kinoyama I, Matsuhisa A, Nakano K, Shishido T, Koutoku H, Sasamata M: YM155, a novel survivin suppressant, enhances taxane-induced apoptosis and tumor regression in a human Calu 6 lung cancer xenograft model. Anti-Cancer Drugs 22: 454-462, 2011 (http://www.ncbi.nlm.nih.gov/pubmed/21389848).

20. Hanahan D and Weinberg RA: The hallmarks of cancer. Cell 100: 57-70, 2000

21. Xia WL, Bisi J, Strum J, Liu L, Carrick K, Graham KM, Treece AL, Hardwicke MA, Dush M, Liao Q, Westlund RE, Zhao S, Bacus S and Spector NL: Regulation of survivin by ErbB2 signaling: therapeutic implications for ErbB2-overexpressing breast cancers. Cancer Res 66: 1640-1647, 2006.

22. Li F: Survivin study: what is the next wave? J Cell Physiol 197: 8-29, 2003.

23. Zhu N, Gu L, Findley HW, Li F and Zhou M: An alternatively spliced survivin variant is positively regulated by p53 and sensitizes leukemia cells to chemotherapy. Oncogene 23: 7545-7551, 2004.

24. Caldas H, Honsey LE and Altura RA: Survivin 2alpha: a novel survivin splice variant expressed in human malignancies. Mol Cancer 4: 11, 2005.

25. Ling $X$, Cheng Q, Black JD and Li F: Forced expression of survivin-2B abrogates mitotic cells and induces mitochondriadependent apoptosis by blockade of tubulin polymerization and modulation of Bcl-2, Bax, and survivin. J Biol Chem 282: 27204-27214, 2007.

26. Vegran F, Boidot R, Oudin C, Defrain C, Rebucci M and LizardNacol S: Association of p53 gene alterations with the expression of antiapoptotic survivin splice variants in breast cancer. Oncogene 26: 290-297, 2007.

27. Span PN, Tjan-Heijnen VC, Heuvel JJ, de Kok JB, Foekens JA and Sweep FC: Do the survivin (BIRC5) splice variants modulate or add to the prognostic value of total survivin in breast cancer? Clin Chem 52: 1693-1700, 2006.

28. McSherry EA, Donatello S, Hopkins AM and McDonnell S: Molecular basis of invasion in breast cancer. Cell Mol Life Sci 64: 3201-3218, 2007.

29. Beslija S, Bonneterre J, Burstein H, Cocquyt V, Gnant M, Goodwin P, Heinemann V, Jassem J, Köstler WJ, Krainer M, Menard S, Petit T, Petruzelka L, Possinger K, Schmid P, Stadtmauer E, Stockler M, Van Belle S, Vogel C, Wilcken N, Wiltschke C, Zielinski CC and Zwierzina H: Second consensus on medical treatment of metastatic breast cancer. Ann Oncol 18: 215-225, 2007. 